\title{
Hallazgos neurofisiológicos de potenciales evocados auditivos en lactantes con antecedente de prematuridad
}

\author{
Ricardo Hidalgo-Gutiérrez ${ }^{1 *}$, Sabino Suárez-Hortiales ${ }^{1}$, Juan A. Giménez-Scherer² y \\ María I. Fraire-Martínez ${ }^{1}$ \\ ${ }^{1}$ Departamento de Neurofisiología; ${ }^{2}$ Unidad de Investigación Médica en Inmunología. Unidad Médica de Alta Especialidad Hospital de Pediatría, \\ Centro Médico Nacional Siglo XXI, Instituto Mexicano del Seguro Social, Ciudad de México, México
}

\begin{abstract}
Resumen
Introducción: Los potenciales evocados auditivos (PEA) son la prueba neurofisiológica más utilizada para evaluar el desarrollo funcional del tronco encefálico auditivo en lactantes; además, permiten evaluar la audición para las frecuencias altas. Métodos: Se llevó a cabo un estudio observacional, descriptivo, transversal y retrospectivo. Se evaluaron los resultados de PEA de 186 lactantes (372 oídos) con antecedente de prematuridad. Se compararon la respuesta bioeléctrica, la morfología, Ia amplitud, las latencias I, III y V, Ios intervalos I-III, III-V y I-V y los umbrales auditivos en dos grupos de prematuros $<32$ y $\geq 32$ semanas de edad gestacional (SEG). Resultados: La respuesta bioeléctrica, las latencias I, III y V y los intervalos I-III y III-V fueron similares en los dos grupos de prematuros de acuerdo con su edad corregida; al compararse, no se encontraron diferencias estadísticamente significativas $(p>0.05)$ para estas variables. La amplitud, la morfología y el intervalo I-V estuvieron más afectados en el grupo de prematuros < 32 SEG $(p<0.05)$. De los 372 oídos evaluados, se encontraron 275 con audición normal y 97 con algún grado de hipoacusia para las frecuencias altas, más frecuente en los pacientes $<32$ SEG ( $p<0.05)$. Conclusiones: El desarrollo funcional del tronco cerebral auditivo fue similar entre los grupos de prematuros y dentro del rango normal para la edad corregida respectiva. La prematuridad produjo un incremento directamente proporcional y estadísticamente significativo en la frecuencia de hipoacusia para las frecuencias altas.
\end{abstract}

Palabras clave: Potenciales evocados auditivos. Prematuridad. Hipoacusia.

\section{Neurophysiological findings of auditory evoked potentials in infants with a history of prematurity}

\section{Abstract}

Background: Auditory evoked potentials (AEPS) constitutes the most commonly used neurophysiological test to assess the functional development of the auditory brainstem in infants and allows the evaluation of hearing for high frequencies. Methods: An observational, descriptive, cross-sectional and retrospective study was conducted. The AEPS results for 186 infants (372 ears) with a history of prematurity were examined. The bioelectrical response, morphology, amplitude, latencies I, III and V, and intervals I-III, III-V and I-V were compared, as well as auditory thresholds between two groups of premature

Correspondencia:

${ }^{*}$ Ricardo Hidalgo-Gutiérrez

E-mail: ricardo.hidalgo.gutierrez@gmail.com

Disponible en internet: 23-03-2020

Fecha de recepción: 24-09-2019 Fecha de aceptación: 14-12-2019 DOI: 10.24875/BMHIM.19000160
Bol Med Hosp Infant Mex. 2020;77(2):76-82

www.bmhim.com 1665-1146/@ 2019 Hospital Infantil de México Federico Gómez. Publicado por Permanyer. Este es un artículo open access bajo la licencia CC BY-NC-ND (http://creativecommons.org/licenses/by-nc-nd/4.0/). 
infants < 32 and $\geq 32$ weeks of gestational age (WGA). Results: The bioelectrical response, latencies I, III and V, and intervals I-III and III-V were similar between the two groups of premature infants according to their corrected age; no statistically significant differences were found $(p>0.05)$ for these variables. The amplitude, morphology, and the I-V interval were more affected in the group of premature infants $<32$ WGA $(p<0.05)$. Of the 372 ears evaluated, 275 showed normal hearing and 97 showed some degree of hearing loss for high frequencies, which was more frequent in patients $<32$ WGA $(p<0.05)$. Conclusions: The functional development of the auditory brainstem was similar between the groups of premature infants and within the normal range for the respective corrected age. Prematurity produced a directly proportional and statistically significant increase in the frequency of hearing loss for high frequencies.

Key words: Auditory evoked potentials. Prematurity. Hearing loss.

\section{Introducción}

Los potenciales evocados auditivos (PEA) son la prueba neurofisiológica más utilizada para evaluar el desarrollo funcional del tronco encefálico auditivo en lactantes y la audición para las frecuencias altas. Estos PEA se pueden registrar desde electrodos colocados en la piel para el análisis de la función del oído de forma no invasiva y las porciones del sistema nervioso activadas por la estimulación acústica ${ }^{1}$. Los PEA se pueden registrar en pacientes prematuros a partir de la semana 25 de edad gestacional $(\mathrm{SEG})^{2}$. Las características neurofisiológicas van cambiando hasta los 2 años de edad, que es cuando termina la maduración de la vía auditiva ${ }^{3}$.

Los PEA proporcionan una evaluación de la integridad neurológica de las vías auditivas hasta el tallo cerebral, así como del umbral auditivo para las frecuencias altas. Esta evaluación se puede utilizar como una prueba auditiva inicial, independientemente de la edad (desde recién nacidos). También se puede usar como una prueba de seguimiento después del cribado con emisiones otoacústicas (como parte del tamiz auditivo) y como paso inicial en una evaluación audiométrica objetiva completa, sobre todo en pacientes con riesgo de afectación de la audición (como aquellos con antecedente de prematuridad) ${ }^{4}$. Los niños prematuros $<32$ SEG tienen mayor riesgo neurológico ${ }^{5}$. Es crucial identificar los trastornos de la audición desde el nacimiento hasta los primeros meses de vida, con la finalidad de realizar una intervención temprana (antes de los 6 meses de edad). La audición se reconoce como un estándar de salud ${ }^{6}$. México, al igual que muchos países, cuenta con un programa de cribado auditivo con técnicas estandarizadas para la evaluación objetiva de la conducción y la audición, como los PEA. Estos estudios se realizan en el Servicio de Neurofisiología Clínica de la Unidad Médica de Alta Especialidad Hospital de Pediatría Dr. Silvestre Frenk Freund, Centro Médico Nacional Siglo XXI (UMAE HP CMN SXXI), Instituto Mexicano del Seguro Social, lo que ha generado una información concentrada sobre los trastornos auditivos. El análisis de los PEA será de gran utilidad para su mejor comprensión y para delinear las mejores disposiciones para su solución.

\section{Métodos}

Se llevó a cabo un estudio observacional, descriptivo, transversal y retrospectivo en el Servicio de Neurofisiología Clínica de la UMAE HP CMN SXXI, Ciudad de México, con previa autorización por los Comités de Investigación y Ética (R-2018-3603-063). Se seleccionaron los estudios de PEA realizados en pacientes de entre 1 día y 2 años de edad corregida, que fueron referidos por el antecedente de prematuridad de julio de 2016 a junio de 2018. Se realizó el análisis de acuerdo con los criterios de evaluación del servicio para la interpretación de los PEA de los pacientes que cumplieron los criterios de inclusión. Los valores obtenidos fueron ajustados para la edad gestacional corregida y comparados con los proporcionados por Halliday ${ }^{7}$, que son los de referencia utilizados en el Servicio de Neurofisiología Clínica. Las variables evaluadas para cada PEA fueron la respuesta bioeléctrica del potencial, la morfología, la amplitud, las latencias, los intervalos y el umbral auditivo. Se realizó el análisis estadístico descriptivo con medidas de tendencia central para la descripción de las variables y se utilizaron las pruebas de ji al cuadrado y exacta de Fisher (según el número de datos) para la comparación de variables nominales y de los grupos por sus diferentes grados de prematuridad, usando el programa SPSS.

\section{Resultados}

Durante el periodo de estudio se realizaron 218 PEA en pacientes que fueron referidos por prematuridad. De estos 218 pacientes, 186 cumplieron los criterios necesarios para el estudio, de los cuales 99 fueron de sexo masculino 
Tabla 1. Comparación de las variables de los potenciales evocados auditivos por grados de prematuridad

\begin{tabular}{|l|c|c|c|c|c|}
\hline \multirow{2}{*}{} & \multicolumn{2}{|c|}{ Prematuros $<32$ SEG } & \multicolumn{2}{|c|}{ Prematuros $\geq 32$ SEG } & P \\
\cline { 2 - 6 } & Normal & Alterada & Normal & Alterada & \\
\hline Respuesta bioeléctrica (N = 372) & $86(95.6 \%)$ & $4(4.4 \%)$ & $276(97.9 \%)$ & $6(2.1 \%)$ & 0.263 \\
\hline Morfología (N = 362) & $77(89.5 \%)$ & $9(10.5 \%)$ & $272(98.6 \%)$ & $4(1.4 \%)$ & $<0.001$ \\
\hline Amplitud (N = 362) & $80(93 \%)$ & $6(7 \%)$ & $271(98.2 \%)$ & $5(1.8 \%)$ & 0.025 \\
\hline Latencia I (N = 362) & $85(98.8 \%)$ & $1(1.2 \%)$ & $273(98.9 \%)$ & $3(1.1 \%)$ & 1.000 \\
\hline Latencia III (N = 362) & $83(96.5 \%)$ & $3(3.5 \%)$ & $273(98.9 \%)$ & $3(1.1 \%)$ & 0.149 \\
\hline Latencia V (N = 362) & $76(88.4 \%)$ & $10(11.6 \%)$ & $260(94.2 \%)$ & $16(5.8 \%)$ & 0.091 \\
\hline Intervalo I-III (N = 362) & $84(97.7 \%)$ & $2(2.3 \%)$ & $268(97.1 \%)$ & $8(2.9 \%)$ & 1.000 \\
\hline Intervalo III-V (N = 362) & $81(94.2 \%)$ & $5(5.8 \%)$ & $269(97.5 \%)$ & $7(2.5 \%)$ & 0.166 \\
\hline Intervalo I-V (N = 362) & $76(88.4 \%)$ & $10(11.6 \%)$ & $264(95.7 \%)$ & $12(4.3 \%)$ & 0.020 \\
\hline
\end{tabular}

SEG: semanas de edad gestacional.

(53.2\%) y 87 de sexo femenino (46.8\%). La mediana de edad gestacional de los 186 pacientes fue de 33 SEG (mínimo de 25 y máximo de 36) y el intervalo intercuartilar fue de 2 SEG. Esto permitió la división de la muestra en dos grupos de acuerdo con el grado de prematuridad: prematuros de < 32 SEG (45 pacientes, $24.2 \%$ ) y prematuros de $\geq 32$ SEG (141 pacientes, 75.8\%). Se evaluó el estudio de PEA de todos los pacientes tomando en cuenta su edad corregida. El intervalo de edad corregida al momento del estudio fue de 1 semana a 18 meses.

\section{Factores de riesgo}

De los 186 pacientes, 161 (86.6\%) presentaron al menos otro factor de riesgo asociado (además de la prematuridad); 25 pacientes (13.4\%) no lo presentaron. Los factores de riesgo más frecuentes fueron hiperbilirrubinemia (72 casos), hemorragia intraventricular (46 casos), sepsis neonatal con uso de ototóxicos (43 casos), asfixia perinatal (35 casos), síndrome de dificultad respiratoria (33 casos) y encefalopatía hipóxico-isquémica (16 casos), entre otros. En algunos casos se presentaron más de un factor de riesgo por paciente.

Por grado de prematuridad, se observó, al menos, un factor de riesgo asociado además de la prematuridad en el grupo de los prematuros de $<32$ SEG $(n=45)$. En el grupo de los prematuros de $\geq 32$ SEG $(n=141)$ se encontraron factores de riesgo asociados en $116(82.3 \%)$, pero ningún otro factor asociado en $25(17.7 \%)$; la diferencia fue significativa $(p<0.05)$.

\section{Fase neurológica}

Cada paciente cuenta con dos vías auditivas, cuya maduración y daño pueden ser independientes entre sí, por lo cual se consideró a cada una como la unidad funcional. Se evaluaron, en total, 372 vías auditivas y se compararon los grupos divididos por grados de prematuridad. Se determinaron la respuesta bioeléctrica (ausencia o presencia del potencial auditivo), la morfología, la amplitud, las latencias absolutas y los intervalos de conducción de estos potenciales para cada vía auditiva. Los oídos con ausencia de respuesta bioeléctrica directamente se catalogaron como hipoacusia profunda y ya no se determinaron las demás mediciones para los diferentes elementos del potencial. En la tabla 1 se agrupan y comparan las variables por grado de prematuridad.

\section{RESPUESTA BIOELÉCTRICA DEL PEA}

De las 372 vías auditivas en que se realizó PEA, se encontró respuesta bioeléctrica en 362 (97.3\%). No se observó respuesta en 10 (2.7\%); esto se refiere a que no se encontró potencial con un estímulo auditivo de $97 \mathrm{~dB}$, que es la intensidad máxima a la que se puede estimular la vía auditiva con el equipo utilizado en el Servicio de Neurofisiología Clínica.

\section{Morfología del PEA}

De las 362 vías auditivas evaluadas, se encontró morfología normal en 349 (96.4\%) oídos y morfología 
Tabla 2. Pacientes por tipo de hipoacusia y su relación con la fase neurológica de los potenciales evocados auditivos

\begin{tabular}{|c|c|c|c|}
\hline & \multicolumn{2}{|c|}{ Fase neurológica } & \multirow[t]{2}{*}{ Total } \\
\hline & Normal & Alterada & \\
\hline $\begin{array}{l}\text { Hipoacusia unilateral }(\mathrm{N}=23 \text { ) } \\
\text { Conductiva } \\
\text { Sensorial } \\
\text { No se puede determinar }\end{array}$ & $\begin{array}{c}12 \\
0 \\
5\end{array}$ & $\begin{array}{l}4 \\
1 \\
1\end{array}$ & $\begin{array}{c}16(26.7 \%) \\
1(1.7 \%) \\
6(10 \%)\end{array}$ \\
\hline $\begin{array}{l}\text { Hipoacusia bilateral ( } \mathrm{N}=37 \text { ) } \\
\text { Conductiva } \\
\text { Sensorial } \\
\text { No se puede determinar } \\
\text { Conductiva/sensorial* } \\
\text { Conductiva/no se puede } \\
\text { determinar* } \\
\text { Sensorial/no se puede } \\
\text { determinar* }\end{array}$ & $\begin{array}{l}11 \\
1 \\
2 \\
1 \\
1 \\
2\end{array}$ & $\begin{array}{l}7 \\
0 \\
6 \\
1 \\
5 \\
\\
0\end{array}$ & $\begin{array}{c}18(30 \%) \\
1(1.7 \%) \\
8(13.3 \%) \\
2(3.3 \%) \\
6(10 \%) \\
2(3.3 \%)\end{array}$ \\
\hline Total & $\begin{array}{c}35 \\
(58.3 \%)\end{array}$ & $\begin{array}{c}25 \\
(41.7 \%)\end{array}$ & $\begin{array}{c}60 \\
(100 \%)\end{array}$ \\
\hline
\end{tabular}

*Pacientes con diferente tipo de hipoacusia entre uno y otro oído.

alterada en $13(3.6 \%)$. En los pacientes prematuros $<32$ SEG se observó una mayor frecuencia de alteraciones en la morfología.

\section{Amplitud del pea}

La amplitud fue normal en 351 (97\%) vías auditivas y disminuida en $11(3 \%)$, en especial entre los prematuros más tempranos, con una diferencia estadísticamente significativa.

\section{LATENCIAS}

Se midieron las latencias de las ondas I, III y V, y se encontraron los siguientes resultados en los 362 oídos evaluados: para la onda I, la latencia fue normal en $358(98.9 \%)$ y prolongada en $4(1.1 \%)$; para la onda III, la latencia fue normal en $356(98.3 \%)$ y prolongada en $6(1.7 \%)$; y para la onda $\mathrm{V}$, la latencia fue normal en $336(92.8 \%)$ y prolongada en $26(7.2 \%)$.

\section{INTERVALOS}

Los intervalos de conducción evaluados fueron I-III, III-V y I-V. De los 362 oídos, para el intervalo I-III resultaron normales $352(97.2 \%)$ y prolongados $10(2.8 \%)$; para el intervalo III-V se encontraron 350 (96.7\%) normales y $12(3.3 \%)$ prolongados; y para el intervalo I-V hubo 340 (93.9\%) normales y 22 (6.1\%) prolongados.

\section{Fase audiológica}

En esta fase se determinó el umbral auditivo de cada oído y, según este, se clasificó una audición normal o hipoacusia para las frecuencias altas $(2000-4000 \mathrm{~Hz})$. Además, por medio de la ecuación de curva latencia/intensidad se estableció el tipo de hipoacusia en la mayoría de los casos. Las vías auditivas que no tuvieron respuesta bioeléctrica en la fase neurológica se catalogaron directamente como hipoacusia profunda, sin determinar el tipo.

\section{UMBRAL AUDITIVO}

Respecto a los umbrales definidos en la muestra, se encontraron 275 oídos (74\%) con audición normal y $97(26 \%)$ con hipoacusia. De estos últimos, en $60(61.9 \%)$ la hipoacusia fue de tipo conductivo y en 7 (7.2\%) fue de tipo sensorial. En los 30 (30.9\%) oídos restantes no se pudo determinar el tipo de hipoacusia por medio de la curva latencia/intensidad. Por grado de prematuridad y de acuerdo con el umbral auditivo de las 90 vías auditivas evaluadas, se encontraron $58(64.4 \%)$ con audición normal y $32(35.6 \%)$ con hipoacusia en el grupo de los prematuros de $<32$ SEG. Por otro lado, en el grupo de los prematuros de $\geq 32$ SEG (282 vías auditivas evaluadas) se hallaron $217(77 \%)$ con audición normal y 65 (23\%) con hipoacusia. Se encontró una mayor frecuencia de hipoacusia en los prematuros de $<32$ SEG $(p<0.05)$ (Fig. 1).

\section{Pacientes con hipoacusia}

De los 97 oídos con hipoacusia (en 60 pacientes), 37 (61.7\%) mostraron hipoacusia bilateral y 23 (23.3\%) unilateral. El intervalo de edad corregida al momento del estudio de los PEA en estos pacientes fue de 1 semana a 14 meses, con $34(56.7 \%)$ pacientes que tuvieron hasta 1 mes y 26 (43.3\%) que fueron mayores de 1 mes, lo que indica una mayor frecuencia de hipoacusia en los pacientes de menor edad corregida.

De los 60 pacientes con hipoacusia, 35 (58.3\%) no mostraron afectación del PEA en la fase neurológica y $25(41.7 \%)$ mostraron afectación unilateral (6) o bilateral (19). La distribución de los pacientes con hipoacusia, el tipo de hipoacusia y su relación con la normalidad o la alteración con la fase neurológica del PEA se observan en la tabla 2.

De los 60 pacientes con hipoacusia, 6 (10\%) carecían de factores de riesgo asociados, además de la prematuridad, y 54 (90\%) presentaban al menos un factor de 


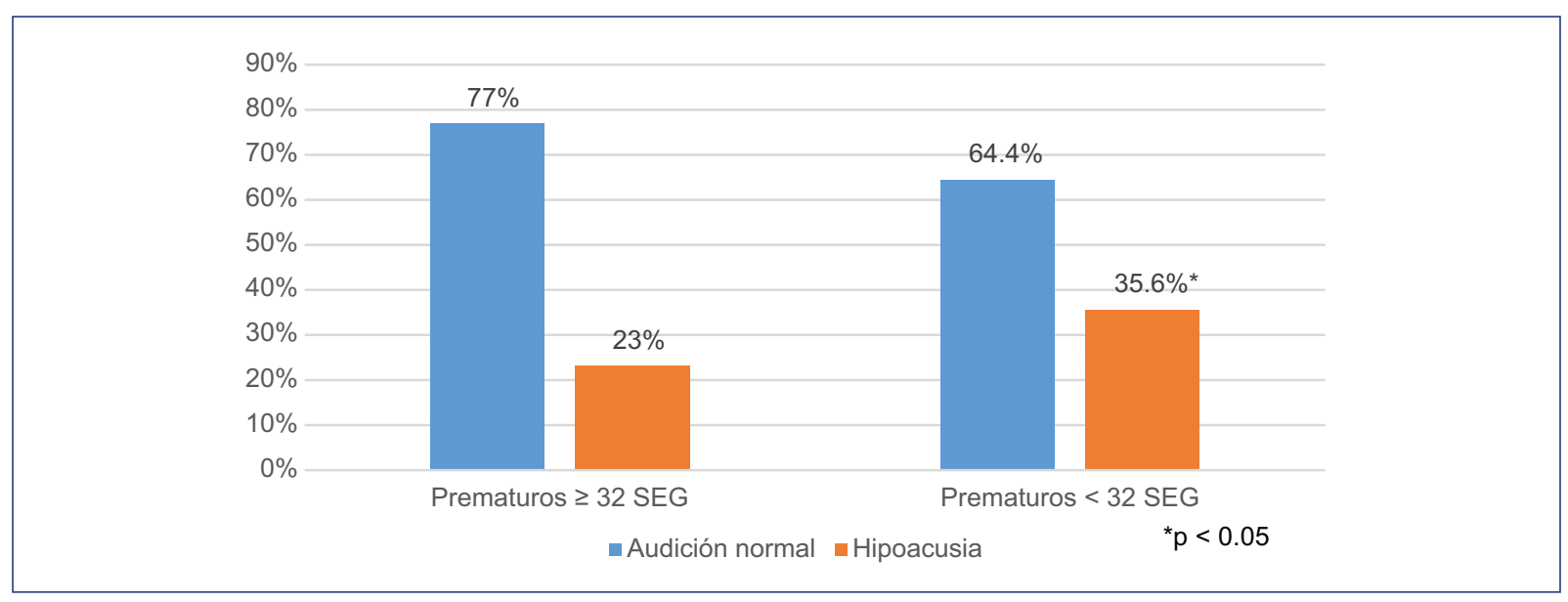

Figura 1. Frecuencia de hipoacusia por grado de prematuridad. SEG: semanas de edad gestacional.

riesgo asociado con la prematuridad. Considerando a los 126 pacientes sin hipoacusia, 19 (15.1\%) no presentaban factores de riesgo asociados con la prematuridad y $107(84.9 \%)$ tenían al menos un factor de riesgo asociado con la prematuridad. La comparación entre los dos grupos no fue estadísticamente significativa $(p>0.05)$.

\section{Discusión}

Los pacientes prematuros constituyen una población de alto riesgo con una gran probabilidad de padecer secuelas neurológicas, entre ellas hipoacusia 0 disfunción de la vía auditiva ${ }^{8}$. Los PEA son una herramienta útil en el tamizaje de las vías auditivas de los pacientes con factores de riesgo como la prematuridad. Los hallazgos encontrados en este estudio se dividieron en dos principales rubros: la fase neurológica y la fase audiológica.

En general, los resultados en la fase neurológica de Ios PEA indican que la conducción a través de la vía auditiva del tronco cerebral en los pacientes con antecedente de prematuridad es normal al evaluarlos de acuerdo con su edad corregida. Por tal motivo, no se encontró diferencia significativa en las latencias I, III y $\mathrm{V}$ ni en los intervalos I-III y III-V cuando se compararon los grupos por grado de prematuridad. La excepción se observó en la medición del intervalo I-V, el cual se encontró prolongado con mayor frecuencia en los pacientes < 32 SEG. Asimismo, se observó una disminución de la amplitud y una alteración de la morfología en el mismo grupo. Cabe destacar que la totalidad de los oídos afectados para estas dos últimas variables durante la fase audiológica se encontraron con hipoacusia, lo que explica su afección.

La probabilidad de que el desarrollo auditivo se produzca con el mismo patrón entre los diferentes grupos de recién nacidos prematuros todavía es desconocida. En la mayoría de los estudios reportados en la literatura se comparan prematuros con recién nacidos a término, y se ha encontrado una mayor afectación en los recién nacidos prematuros que en los recién nacidos a término, sobre todo en las latencias, lo que sugiere como principal factor biológico la diferencia del grado de mielinización ${ }^{9-12}$. En los estudios mencionados, los PEA anormales podrían deberse a la influencia de complicaciones neonatales 0 a problemas perinatales en comparación con los controles, en lugar de solo a la prematuridad como factor de riesgo. Es interesante que la mayoría de los pacientes prematuros reclutados en estos estudios presentaban otros factores de riesgo asociados, además de la prematuridad, lo que puede contribuir a demorar el proceso de maduración de la vía auditiva. Por tal motivo, se sospecha que existen otros factores que pudieron influir en los pacientes más prematuros en este estudio. Existen reportes en los que no se observaron diferencias en Ios PEA entre recién nacidos prematuros y recién nacidos a término, siempre que la edad gestacional fue corregida ${ }^{13}$.

Los únicos estudios que describen los hallazgos de Ios PEA en los diferentes grados de prematuridad, comparándolos entre ellos, son los de Seethapathy et al. ${ }^{14,15}$, quienes encontraron una afectación similar en los diferentes grados de prematuridad para todas las variables evaluadas una vez corregida la edad 
gestacional, incluyendo latencias, intervalos y amplitudes. No compararon la morfología, debido a que esta variable no se incluyó en su estudio. Ya que los resultados obtenidos en la fase neurológica en los PEA del presente estudio indican que la función auditiva del tronco cerebral en diferentes grupos de prematuros es similar si se evalúa de acuerdo con su edad corregida, la corrección de la edad gestacional mitiga los efectos de la madurez biológica sobre el desarrollo neurológico al normalizar los valores de referencia utilizados para calificar los resultados. Por lo tanto, las alteraciones más acentuadas en el intervalo I-V, la amplitud y la morfología de los PEA a una edad corregida pueden estar relacionadas con la edad gestacional de los recién nacidos prematuros, y ser solo un dato de inmadurez de la vía auditiva en este grupo de pacientes.

La fase audiológica de los PEA reportó resultados más interesantes, ya que el $26 \%$ de las vías auditivas evaluadas mostraron algún grado de hipoacusia, en particular en los pacientes con mayor grado de prematuridad, quienes resultaron los más afectados. Los oídos de 60 pacientes (32.2\% de la muestra total) presentaron hipoacusia, ya fuera bilateral (37 pacientes, $61.7 \%$ ) o unilateral (23 pacientes, $23.3 \%$ ). Esta cifra es mucho mayor que la reportada por la Organización Mundial de la Salud (OMS), que considera que la sordera congénita afecta a 1-3 de cada 1000 neonatos y es la causa de discapacidad neurosensorial más común en el recién nacido ${ }^{16}$. Esta cifra se incrementa a 2-4 por cada 1000 recién nacidos vivos que requirieron hospitalización en la unidad de cuidados intensivos neonatales ${ }^{17}$.

Aunque la OMS considera la prematuridad como uno de los factores de riesgo más importantes para la hipoacusia congénita no genética, no reporta cifras de incidencia de hipoacusia en pacientes prematuros. Un estudio realizado por Hernández-Herrera et al. ${ }^{18}$ con población mexicana reportó una frecuencia de hipoacusia del $5 \%$ en neonatos con factores de riesgo, incluyendo la prematuridad como uno de ellos. En este estudio se encontró una considerable mayor frecuencia.

Los PEA se han convertido en una herramienta útil en el tamiz auditivo neonatal, ya que la hipoacusia en niños debe detectarse durante el periodo prelingüístico para evitar trastornos de la comunicación. Al presente día, la investigación relacionada con el desarrollo de la vía auditiva en los diferentes grados de prematuridad es escasa, por lo que este estudio ayuda a comprender el desarrollo y la maduración de la vía auditiva en lactantes con antecedente de prematuridad.
Según los hallazgos de este estudio evaluados de acuerdo con la edad gestacional corregida, la respuesta bioeléctrica, la morfología, la amplitud, las latencias y los intervalos de conducción de los PEA en los pacientes con antecedentes de prematuridad, con o sin factores de riesgo asociados, no mostraron diferencias estadísticamente significativas entre los distintos grupos de prematuros y se mantuvieron dentro de los valores de referencia internacionales para las edades gestacionales correspondientes. Este estudio permite llegar a la conclusión de que la prematuridad no parece tener un efecto deletéreo manifiesto en el desarrollo neurológico de los PEA. Por lo tanto, el desarrollo funcional del tronco cerebral auditivo fue similar en los dos grupos de prematuros y dentro del rango normal para la edad corregida respectiva de cada paciente. Sin embargo, se encontró que la prematuridad produjo un incremento directamente proporcional y estadísticamente significativo en la presencia de hipoacusia para las frecuencias altas, afectando a uno de cada tres pacientes de este estudio.

\section{Responsabilidades éticas}

Protección de personas y animales. Los autores declaran que para esta investigación no se han realizado experimentos en seres humanos ni en animales.

Confidencialidad de los datos. Los autores declaran que han seguido los protocolos de su centro de trabajo sobre la publicación de datos de pacientes.

Derecho a la privacidad y consentimiento informado. Los autores han obtenido el consentimiento informado de los pacientes o sujetos referidos en el artículo. Este documento obra en poder del autor de correspondencia.

\section{Conflicto de intereses}

Los autores declaran no tener ningún conflicto de intereses.

\section{Financiamiento}

Ninguno.

\section{Bibliografía}

\footnotetext{
1. Legatt $A D$. Brainstem auditory evoked potentials: methodology, interpretation, and clinical application. En: Aminoff MJ, editor. Aminoff's electrodiagnosis in clinical neurology. California, USA: Elsevier Saunders; 2012. p. 519-52.

2. Starr A, Amlie RN, Martin WH, Sanders S. Development of auditory function in newborn infants revealed by auditory brainstem potentials. Pediatrics. 1977;60:831-9.

3. Eggermont JJ, Salamy A. Maturational time course for the ABR in preterm
} and full term infants. Hear Res. 1988;33:35-47. 
4. Guía de práctica clínica. Hipoacusia neurosensorial bilateral e implante coclear. Consejo de Salubridad General. México: Instituto Mexicano de Seguro Social; 2010.

5. Ramos-Sánchez I, Mázquez-Luque A. Recién nacido de riesgo neurológico. Vox Paediatrica. 2000;8:5-10.

6. Organización Mundial de la Salud. Sordera y pérdida de la audición. OMS; 2019. Disponible en: http://www.who.int/es/news-room/fact-sheets/ detail/deafness-and- hearing-loss.

7. Halliday AM. Evoked potentials in clinical testing. En: Evoked potentials in pediatrics. New York: Churchill Livingstone; 1992. p. 489-521.

8. American Speech-Language-Hearing Association (ASHA). Pérdida de la audición infantil. Rockville: ASHA; 2016. Disponible en: http://www.asha. org/uploadedFiles/AIS-Hearing-Loss-Childhood-Spanish.pdf.

9. Sleifer P, Selaimen da Costa S, Luiz Cóser P, Zubaran Goldani M Dornelles $\mathrm{C}$, Weiss $\mathrm{K}$. Auditory brainstem response in premature and full-term children. Int J Pediatr Otorhinolaryngol. 2007;71:1449-56.

10. Ribeiro FM, Carvallo RM. Tone-evoked ABR in full-term and preterm neonates with normal hearing. Int J Audiol. 2008:47:21-9.

11. Casali RL, Santos MF. Auditory brainstem evoked response: response patterns of full-term and premature infants. Braz $\mathrm{J}$ Otorhinolaryngol. 2010;76:729-38.
12. Jiang ZD, Ping LL. Functional integrity of rostral regions of the immature brainstem is impaired in babies born extremely preterm. Clin Neurophysiol. 2016;127:1581-8.

13. Kaga K, Hashira S, Marsh RR. Auditory brainstem responses and behavioural responses in pre-term infants. Br J Audiol. 1986;20:121-7.

14. Seethapathy J, Boominathan P, Uppunda AK, Ninan B. Auditory brainstem response in very preterm, moderately preterm and late preterm infants. Int J Pediatr Otorhinolaryngol. 2018;111:119-27.

15. Seethapathy J, Boominathan P, Uppunda AK, Ninan B. Changes in auditory brainstem response in very preterm and late preterm infants. Int $\mathrm{J}$ Pediatr Otorhinolaryngol. 2019;121:88-94.

16. Organización Mundial de la Salud. Pérdida de audición en la niñez. Ginebra: OMS; 2016. Disponible en: http://www.who.int/topics/deafness/ WHD brochure ES.pdf?ua=1.

17. Castellanos-Coutiño M, Santamaría-Muñoz R, Escobar-Carrillo M. Hipoacusia mediante emisiones otoacústicas en el recién nacido de la UCIN. Salud Tabasco. 2012;18:45-9.

18. Hernández-Herrera RJ, Hernández-Aguirre LM, Castillo-Martínez NE, de la Rosa-Mireles N, Martínez-Elizondo J, Alcalá-Galván LG, et al. Tamizaje y confirmación diagnóstica de hipoacusia. Neonatos de alto riesgo versus población abierta. Rev Med IMSS. 2007;45:421-6. 